\title{
Germanica
}

\section{Der tragische Held des Realismus}

Zur Rezeption von Louis Aragon in der DDR

Le héros tragique du réalisme. Sur la réception de Louis Aragon en RDA

The tragic hero of Realism. On the reception of Louis Aragon in the GDR

\section{Florian Gödel}

\section{OpenEdition}

\section{Journals}

Édition électronique

URL : http://journals.openedition.org/germanica/3547

DOI : 10.4000/germanica.3547

ISSN : 2107-0784

\section{Éditeur}

Université de Lille

\section{Édition imprimée}

Date de publication : 30 décembre 2016

Pagination : 161-170

ISBN : 9782913857384

ISSN : 0984-2632

Référence électronique

Florian Gödel, „Der tragische Held des Realismus“, Germanica [Online], 59 | 2016, Online erschienen am 30 Dezember 2018, abgerufen am 06 Januar 2021. URL: http://journals.openedition.org/germanica/ 3547 ; DOI: https://doi.org/10.4000/germanica.3547 


\title{
Der tragische Held des Realismus. Zur Rezeption von Louis Aragon in der DDR.
}

\author{
Florian GöDEL \\ Friedrich-Schiller-Universität Jena
}

Louis Aragon begleitet die DDR von Anfang bis Ende. Sie rezipiert ihn wie kaum einen anderen französischen Dichter während des gesamten Zeitraums ihres Bestehens. Aragon erscheint als ein Schriftsteller mit drei Schaffensphasen: einer avantgardistischen (Dada und Surrealismus), einer sozialistisch-realistischen (mit der Résistance) und schließlich einer hier als experimental-realistisch bezeichneten Phase. Dennoch wird in der DDR der Anspruch erhoben, den ganzen Dichter Aragon zu fassen.

Unabhängig von seinen poetologischen Überzeugungen wird er in diesen rund 40 Jahren fast gleichbleibend stark rezipiert. Allerdings lässt sich an der Entwicklung der Auflagenhöhe seiner Werke eine deutliche Tendenz ausmachen: Die Höhe der Erstauflagen sinkt ab 1962 signifikant ab, während die vorher publizierten Werke aus der Phase des sozialistischen Realismus kontinuierlich in hoher Zahl nachgedruckt werden ${ }^{1}$. Der Bruch liest sich deutlich ab am Übergang von der Karwoche (La Semaine sainte 1958/1962) ${ }^{2}$ zu den Spiegelbildern

1. - Siehe hierzu: Danielle Risterucci-Roudnicky, France - RDA. Anatomie d'un transfert littéraire 1949 - 1990, Bern u.a., Peter Lang, 1999, S. 151.

2. - Die erste Datumsangabe beschreibt, wenn nicht anders angegeben, den Zeitpunkt der Erstveröffentlichung des französischen Originals, die zweite Angabe steht für die Erstveröffentlichung in der DDR. 
(La Mise à mort 1965/1968). Erstere erscheint bei Volk und Welt in einer Auflagenhöhe von 15.000 Exemplaren. Die Spiegelbilder als der erste Roman des Spätwerks sollen ursprünglich ebenfalls in 15.000 Exemplaren erscheinen, allerdings bittet hier Volk und Welt schon bald um eine drastische Verringerung auf 3.000 Exemplare ${ }^{3}$. Dies erscheint umso erstaunlicher, als man Gallimard, Aragons Stammverlag, bereits eine Lizenz in Höhe von 10.000 Exemplaren abgekauft hatte. Auch die anschließenden Publikationen des Spätwerkes und dann der avantgardistischen Phase kamen nicht mehr über 6.000 Exemplare hinaus. Gemessen an den Verhältnissen der DDR war dies wenig. De facto wurde Aragon hier also nicht anders behandelt als die restliche französische Moderne. Zudem wurden ab Theater/Roman (1974/1978) alle Neuveröffentlichungen, d.h. auch das avantgardistische Frühwerk, als Lizenz von westdeutschen Verlagen übernommen.

Damit sind schon die zwei zentralen Zeitpunkte für die DDR-Rezeption benannt: Einerseits 1962 als der Übergang vom sozialistischen Realismus zum experimentellen Realismus ${ }^{4}$ und andererseits 1977-1978 als der offizielle Rezeptionsbeginn des Frühwerkes. Die Druckgenehmigungsverfahren zu den beiden entsprechenden Werken, Spiegelbilder und Anicet oder das Panorama, Roman (Anicet ou le panorama, roman 1921/1978), sollen deshalb später detailliert besprochen werden, auch weil die DDR-Zensur rezeptionsgeschichtlich von ganz eigener Wichtigkeit ist.

Der Artikel bedient sich zudem zweier inhaltlicher Parameter, anhand derer die Rezeptionsgeschichte von Aragons Werk besser nachvollzogen werden kann. Zum ersten ist das die Kategorie des Spiels, unter die verschiedene Elemente des Aragon-Bildes zusammengefasst werden können, etwa die Intellektualität seiner Persönlichkeit, die Komplexität seiner Texte und daraus resultierend die Ausrichtung auf die Suche bzw. das Experiment. Zum zweiten ist das die Kategorie der Liebe, insbesondere im Zusammenhang mit Aragons SchriftstellerGattin Elsa Triolet und dem immer wieder aufgenommenen Zitat: „Die Zukunft des Menschen ist die Frau ${ }^{5}$ “.

Da es nicht möglich war, eine vollständige Rezeptionsgeschichte vorzulegen, liegt dem Artikel eine historische Übersicht der verwendeten Primärtexte bei.

3. - Bundesarchiv, Akte DR 1/2335, pag. 66, 69.

4. - Der Ausdruck stützt sich auf einen Aufsatz des Autors, in dem er ein „Bürgerrecht für einen experimentellen Realismus“ fordert. Louis Aragon, „Das Ende der ,Wirklichen Welt"“, in: Karlheinz Barck und Brigitte Burmeister (Hrsg.), Ideologie - Literatur - Kritik. Französische Beiträge zur marxistischen Literaturtheorie, Berlin, Akademie-Verlag, 1977, S. 242.

5. - U.a. Louis Aragon, Zu lieben bis Vernunft verbrennt. Gedichte, Hrsg. und mit einem Nachwort versehen v. Marianne Dreifuß, Berlin, Volk und Welt, 1968. S. 174. 


\section{Die Anfänge - Leitbild Résistance-Dichter}

Mit dem Aragon-Bild der DDR der 1950er Jahre verbunden ist vor allem seine Rolle als brillanter Intellektueller, als treuer Kommunist und als genialer Schriftsteller. Der Kämpfer der politischen Avantgarde scheint die ästhetische Komponente weitgehend zu verdecken. Schon 1946 wendet sich Stephan Hermlin in einem offenen Brief an Aragon und bittet ihn um eine Aussöhnung mit den schuldbeladenen Deutschen, wobei Hermlin den kommunistischen Widerstand in Deutschland ausdrücklich in diese Schuld, „das Regime nicht gestürzt zu haben6“, miteinbezieht. Aragon erscheint hier als der glanzvolle Held einer in allen Belangen vorbildlichen französischen Résistance. In einem zweiten Brief zu Aragons 60. Geburtstag nennt ihn Hermlin unumwunden den größten lebenden französischen Dichter ${ }^{7}$. Aragon passt ausgezeichnet in Johannes R. Bechers und Walter Ulbrichts dominierende Vorstellung von der DDR als der Vollendung des goethezeitlichen Humanismus ${ }^{8}$. Hans Mayer, federführend in der frühen Aragon-Rezeption, wichtiger Übersetzer, Wissenschaftler und Begutachter seines Werkes, beschreibt in seinem Nachwort zur Karwoche Aragon als den historisch reflektierten Erben des goetheschen Begriffs von der Menschheit als etwas, das mehr ist als die Summe der Individuen, und grenzt ihn stark von Sartres angeblich unhistorischem und individualistischem Freiheitsbegriff $a^{9}{ }^{9}$. Aragon fungiert somit auch als eine zentrale Gestalt in der Debatte um eine spezifisch sozialistische Annahme des nationalen Erbes und seiner Befreiung aus der nationalsozialistischen Vereinnahmung. In dieses frühe Bild passt die vermeintlich düstere surrealistische Vergangenheit schlecht hinein. Hans Mayer versucht anfangs sogar noch, den Surrealisten gänzlich zu verschweigen ${ }^{10}$, muss aber schon bald einsehen, dass in einer DDR, die den Anspruch erhebt, den ganzen Aragon fassen und erklären zu wollen, ein Weg gefunden werden muss, den Surrealismus zu integrieren. Das Ergebnis zeigt sich bald: Seit den 1960er Jahren findet sich in beinahe jedem Gutachten, Geleitwort und Artikel zu Aragon die stereotype Phrase, dass dies oder

6. - Stephan Hermlin, „Offener Brief an Aragon“, in: Ders., Äußerungen 1944 1982, Berlin/Weimar, Aufbau, 1983, S. 8.

7. - Stephan Hermlin, „Ein Gruß für Aragon. Zum 60. Geburtstag“, in: ebd., S. 253.

8. - Vgl. Alexander Abusch, ,Wir bewahren Schillers humanistisches Erbe für die ganze Nation“, in: Elimar Schubbe (Hrsg.), Dokumente zur Kunst-, Literatur- und Kulturpolitik der SED, Stuttgart, Seewald, 1972, S. 590.

9. - Hans Mayer, ,Nachwort“, in: Louis Aragon, Die Karwoche, Berlin, Volk und Welt, 1962, S. 673.

10. - So ist noch in Mayers Nachwort zu seiner Übersetzung von Louis Aragon, Herrn Duvals Neffe, Berlin, Volk und Welt, 1955. S. 175 ff. nicht im Mindesten von Aragons surrealistischer Vergangenheit die Rede. 
jenes Werk in Kontinuität zum gesamten Schaffen des Autors gesehen werden müsse. Das heißt zunächst einmal, dass man von einer dialektischen Aufhebung des Surrealismus im sozialistischen Realismus spricht.

\section{Aragons, Wende' und die folgenden Jahrzehnte}

Anfang der 1960er Jahre ändert sich die kulturpolitische Situation in der DDR und auch die Rezeption Aragons grundsätzlich. Aragons Prager Rede von 1962 erweist sich als regelrechter Skandal. Sie wird in der letzten Ausgabe von Sinn und Form veröffentlicht, die Peter Huchel noch zu verantworten hat. In ihr erläutert Aragon seine Utopie eines zukünftigen, Theorie und Praxis vereinenden, ,offenen Realismus ${ }^{11 “ .}$ Daraus folgt eine experimentellere Ausrichtung seiner eigenen realistischen Poetologie als dichterisches Spiel und Suche nach der verlorengegangenen Form für die Wirklichkeit. Diese Reflexionen sind dann direkter Gegenstand seiner Werke. Sie sind Literatur über das Schreiben von Literatur, über den Autor, der diese Literatur hervorbringt. Es ist dabei nur konsequent - und dies wird ihm in der DDR der 1960er Jahre ausdrücklich zum Vorwurf gemacht - dass er sich in die Nähe von Roger Garaudy begibt und für dessen umstrittenes Buch D'un réalisme sans rivages ein begeistertes Vorwort schreibt ${ }^{12}$.

1962 markiert darüber hinaus eine Verlagerung der Debatte von den Zeitschriften wie Sinn und Form und Aufbau auf andere Medien. Besonders stark beginnt die Auseinandersetzung mit Aragon in der Forschung in der zweiten Hälfte der 1970er Jahre in der Fachzeitschrift Beiträge zur Romanischen Philologie (BRPh), die rund um seinen 80. Geburtstag 1977 eine ganze Reihe von Artikeln veröffentlicht. Der Autor wird allmählich selbst Geschichte. Vor allem die Leipziger Romanistin Brigitte Hocke setzt sich in den BRPh mit Aragons Realismusbegriff auseinander ${ }^{13}$. Sie stellt die These auf, dass Aragon einen falschen, weil sensualistischen Widerspiegelungsbegriff vertreten habe, sodass sein Verständnis vom sozialistischen Realismus in die Krise geraten musste ${ }^{14}$. Das Spätwerk erscheint ihr als ein Versuch, wieder an die Wirklichkeit heranzukommen, der jedoch an Aragons Verzweiflung über die triste französische Realität gescheitert sei. Die Vorstellung vom Schriftsteller als einem Opfer seiner gesellschaftlichen Umstände findet

11. - Louis Aragon, ,Rede in Prag“, Sinn und Form, 1962, H. 5-6, S. 928.

12. - Roger Garaudy, D’un réalisme sans rivages. Picasso, Saint-John Perse, Kafka. Préface d'Aragon, Paris, Plon, 1963.

13. - Brigitte Hocke, „Über die Grenzen von Aragons Realismus-Begriff (Zur Neufassung des Romans Les Communistes)“", Beiträge zur Romanischen Philologie XVI/ 1977, H. 2, S. $219 \mathrm{ff}$.

14. - Vgl. ebd., S. 238 f. 
sich wesentlich später in Rita Schobers Publikation Louis Aragon. Von der Suche der Dichtung nach der Erkenntnis der Welt ${ }^{15}$ wieder und wird als Topos der tragischen Suche zu einem Grundmuster der Interpretation seines Schreibens erhoben. Diese Begründung eines Scheiterns an historisch-nationalen Umständen ist gleichzeitig ein Versuch, die eigenen Dichter in der DDR von der Notwendigkeit einer Nachahmung freizusprechen. Nur Irene Selle macht in einem ihrer Beiträge in den $B R P h$ direkt den Charakter des Schriftstellers für diese Entwicklung verantwortlich. Sie bescheinigt ihm wenig feinfühlig einen ,antidogmatische[n] Komplex ${ }^{16 “ .}$. Der Vorwurf zielt wieder auf die Grundkategorie des Spiels, die hier negativ im Sinne des surrealistischen Frühwerks verstanden wird. Trotzdem ist es auffällig, dass in der Forschung allgemein ein freierer Ton herrscht als in den anderen Rezeptionsgebieten. Höhepunkte sind hier unter anderem der Beitrag

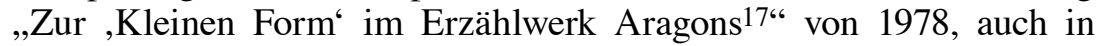
den $B R P h$ erschienen, verfasst von Gerhard Schewe, der als externer Gutachter neben Rita Schober die größte Rolle spielt und ebenso wie sie Nachworte verfasst. Neue Maßstäbe in der Bewertung des Surrealisten setzt Michael Nerlich in seinem Artikel „Für ein geschichtliches Verständnis des ,Surrealismus'. Anmerkungen zum Frühwerk Aragons“ von 197718. Nerlich grenzt Aragon von Breton ab. Allerdings argumentiert er nicht für eine ,Freisprechung ' Aragons vom Surrealismus - das hatten früher auf ähnliche Weise schon andere getan -, sondern für eine Differenzierung der surrealistischen Strömung in ihrer Gesamtheit. Allerdings tauchen auch in der Forschung immer wieder Stimmen auf, die überkommene ideologische Schablonen kopieren. Als Beispiel sei hier Helmut Melzers „Kampf für den Frieden und die künstlerische Entwicklung. Der Beitrag Louis Aragons“ (1984) genannt ${ }^{19}$. Melzer differenziert überhaupt nicht zwischen künstlerischen und politischen Aspekten. Dagegen setzt sich in den 1980er Jahren eine zunehmend entpolitisierte Sprache durch. L. Soninas Artikel „Aragons

15. - Rita Schober, Romanistin in Berlin, ist eine zentrale Gestalt in der späteren wissenschaftlichen Aragon-Rezeption der DDR. Rita Schober, Louis Aragon. Von der Suche der Dichtung nach der Erkenntnis der Welt, Berlin, Akademie-Verlag, 1985.

16. - Irene Selle, „Aragons Realismuskonzeption im Lichte seiner Erbetheorie“, Beiträge zur Romanistischen Philologie XVII/ 1978, H. 1, S. 57.

17. - Gerhard Schewe, ,Zur ,Kleinen Form“ im Erzählwerk Aragons“, Beiträge zur Romanischen Philologie XVII/ 1978, H. 1. S. 58-63.

18. - Michael Nerlich, „Für ein geschichtliches Verständnis des ,Surrealismus“: Anmerkungen zum Frühwerk Aragons“", Beiträge zur Romanische Philologie XVI/ 1977, H. 1, S. $83 \mathrm{ff}$.

19. - Helmut Melzer, „Kampf für den Frieden und künstlerische Entwicklung Der Beitrag Louis Aragons“, Beiträge zur Romanischen Philologie XXIII/1984, H. 1, S. $63 \mathrm{ff}$. 
,Metaphernroman““, der 1985 in der sowjetwissenschaftlichen Fachzeitschrift Kunst und Literatur erscheint, bringt zum ersten und einzigen Mal Aragon in eine namentliche Nähe zum Nouveau Roman und benennt als Ursache für seine Annäherung an dessen darstellerische Mittel eine Aktualitätssucht, die den Dichter dazu getrieben habe, immer wieder das literarische Tagesgespräch zu dominieren ${ }^{20}$. Allerdings habe Aragons Festhalten an einer ,,anthropozentrisch[en]" Grundhaltung ein tieferes Übereingehen mit den Nouveaux Romanciers verunmöglicht ${ }^{21}$. Die entpolitisierte Sprache setzt sich sogar in den Gutachten durch, sodass Brigitte Hocke 1988 im externen Gutachten zur Neufassung der Kommunisten fordern kann, dass sich die ästhetischen Fragestellungen an den Autor vollkommen emanzipieren sollten von der politischen Rolle, die er eingenommen hat ${ }^{22}$. Auch hier erscheint die Historisierung offenkundig. Aragon ist zu diesem Zeitpunkt bereits sechs Jahre tot.

\section{Rezeptionslücke Lyrik}

Bevor zu den Gutachten der maßgeblichen Romanwerke übergegangen werden soll, muss noch die Rezeption von Aragons Lyrik angesprochen werden. Im DDR-Bild des Autors ist sie extrem unterrepräsentiert. Es wird nur eine einzige Anthologie mit Gedichten veröffentlicht. Dabei handelt es sich um $\mathrm{Zu}$ lieben bis Vernunft verbrennt von 1968, herausgegeben von Marianne Dreifuß, die als FranzösischLektorin bei Volk und Welt vor allem in den 1950er Jahren eine große Rolle gespielt hat. Später greift sie oft noch als Gutachterin in die Druckgenehmigungsverfahren des Verlages ein. Der Band setzt einen klaren Schwerpunkt auf Aragons Résistance-Lyrik, was nicht verwundert im Hinblick auf die offenbar größeren Schwierigkeiten, überhaupt Lyrik von ihm eigenständig zu publizieren. Dreifuß fokussiert Aragons Liebesbekundungen an Elsa Triolet, die eine essentielle Stellung in seiner Lyrik überhaupt und auch in der Prosa einnimmt ${ }^{23}$. Die dichterische Gestaltung der Liebe zu einer emanzipierten Kommunistin, die in der FKP von nicht geringer Bedeutung ist, wird von Dreifuß und später von Schober und Hocke als Beweis für die Bekehrung vom Sündenfall des Surrealismus interpretiert ${ }^{24}$. Vor dem Hintergrund der Liebe zu Elsa Triolet ist es jedoch erstaunlich, dass die gleiche Marianne Dreifuß

20. - L. Sonina, „Aragons ,Metaphernroman““, Kunst und Literatur. Sowjetwissenschaften, Jg. 33, Januar/ Februar 1985, H. 1, S. $71 \mathrm{ff}$.

21. - Ebd., S. 83.

22. - Vgl. Bundesarchiv, Akte DR 1/2391, pag. 105.

23. - Einige Werke, die hierfür in Frage kommen, sind Les Yeux d'Elsa, Elsa, Le Fou d'Elsa.

24. - Bei Schober wird dieser Bekehrungsvorgang fast wörtlich genommen. Vgl. Rita Schober, Louis Aragon..., a.a.O., S. 156. 
in einem Gutachten zu Spiegelbilder im selben Jahr schreibt, der Roman sei eine Anklage gegen die „Göttin Elsa“, die in Aragon nur den berühmten Kommunisten liebe 25 .

\section{Druckgenehmigungsverfahren 1: Spiegelbilder von 1968}

Spiegelbilder erscheint 1968, drei Jahre nach dem französischen Original La Mise à mort, bei Volk und Welt. Schon der deutsche Titel betont gewissermaßen die Brisanz des Buches für einen neuen Widerspiegelungsbegriff in Aragons Werk. Das Druckgenehmigungsverfahren erweist sich dementsprechend als kompliziert. Den Akten liegt zuallererst ein Brief des Verlags bei, der besonders die Kunstfertigkeit, die Zeit- und die Selbstkritik des Autors lobt ${ }^{26}$. Das Verfahren beinhaltet insgesamt drei umfangreiche Gutachten. Zwei internen Verlagsgutachten von Marianne Dreifuß und Klaus Möckel steht ein externes literaturwissenschaftliches Gutachten von Rita Schober gegenüber. Letztere verfasst auch das als Essay angelegte längere Nachwort mit dem Titel „Im Spiegel Fougères oder ,Die Weise von Liebe und Tod"“. Es wird 1974 noch einmal gesondert in den $B R P h$ publiziert ${ }^{27}$. Das Gutachten von Klaus Möckel beginnt mit dem Angebot, dass man den Roman in einer einmaligen Auflage in geringer Zahl drucken könnte, d.h. hier ist man bereits von dem ursprünglichen Plan einer Auflagenhöhe von 15.000 Exemplaren abgerückt ${ }^{28}$. Dann geht Möckel aber in die Offensive über. Die Veröffentlichung sei nicht nur eine Devisenfrage - immerhin verwaltet Volk und Welt die gesamtdeutschen Rechte an Aragons Werk, und Möckel betont die guten Kontakte zum Autor auch über den Willen von Gallimard hinweg -, sondern darüber hinaus sogar ein Politikum. In der suggerierten Dringlichkeit der Situation wählt er einen beinahe staatstragenden Ton:

Würde der Verlag Volk und Welt, und damit die DDR, auf das Werk verzichten, [...] so wäre das ein Anlass für die westdeutsche Presse, eine neue politische Sensation zu schaffen. [...] [N]ach wie vor gehört Aragon zu den führenden politischen Kräften seiner Partei und hat gerade in der vergangenen Wahlperiode eine positive Rolle gespielt ${ }^{29}$.

Ähnlich wie Möckel argumentiert auch Dreifuß vor allem kulturpolitisch. Beiden Gutachtern merkt man an, dass sie ihre Not

25. - Bundesarchiv, Akte DR 1/2335, pag. 76.

26. - Ebd. S. 67.

27. - Rita Schober, „Im Spiegel Fougères oder ,Die Weise von Liebe und Tod““, Beiträge zur Romanistischen Philologie XIII/ 1974, H. 1/2, S. $161 \mathrm{ff}$.

28. - Dessen ungeachtet erscheinen in der DDR noch zwei Nachdrucke: 1974, möglicherweise im Zusammenhang mit dem Essay von Schober in den BRPh, und 1980.

29. - Bundesarchiv, Akte DR 1/2335, pag. 73. 
damit haben, bestehende literarische Beschreibungskategorien auf den ,neuen Aragon' anzuwenden. Hierfür spricht schon der erste Satz von Dreifuß: „Dieses Buch ist so unkonventionell, daß man unmöglich ein Gutachten darüber schreiben kann [... $]^{30 “ " . ~ I m ~ U n t e r s c h i e d ~ z u ~ d e n ~ b e i d e n ~}$ anderen Gutachten argumentiert Schober inhaltlicher. Dass Aragon selbst- und gattungsreflektiert schreibt, erscheint ihr als Ausdruck für einen Realismus höherer Stufe, der notwendigerweise neue literarische Mittel erfordere. So wird argumentiert, dass Aragon von diesen neuen Mitteln Gebrauch machen darf, weil er auf der höchsten erkenntnistheoretischen Stufe steht: Er ist Kommunist. Insofern wird das aragonsche Spiel in der neuen Form eines experimentellen Realismus unter dem Vorbehalt akzeptiert, dass man es später als gescheitertes Experiment in einer kapitalistischen Gesellschaft hinstellen kann ${ }^{31}$. Stark gemacht wird die Argumentationsstrategie des Schriftstellers als eines Zeugen dafür, wie man im kapitalistischen Ausland realistisch schreiben kann, besonders von Dietrich Simon in seinem Gutachten zu Theater/Roman (Théâtre/Roman 1974/1978) ${ }^{32}$. Der Autor habe gewissermaßen ein Recht auf Veröffentlichung kraft seines Zeugenstatus. Aragon wird folglich zum tragischen Helden stilisiert, der die ideologischen Überzeugungen seiner Genossen in den sozialistischen Staaten teile, der aber seinen Realismusbegriff zwangsläufig ausdehnen müsse, wenn er ihn nicht aufgeben wolle.

Diese Strategien taugen freilich nicht in den Druckgenehmigungsverfahren $\mathrm{zu}$ Aragons dadaistisch-surrealistischem Frühwerk, das ab 1978 vorwiegend in der Werkausgabe bei Volk und Welt erscheint.

\section{Druckgenehmigungsverfahren 2: Anicet oder das Panorama, Roman von 1978}

Anicet oder das Panorama, Roman erscheint 1978, rund 57 Jahre nach der französischen Erstveröffentlichung in der DDR. Gerhard Schewe schreibt schon 1974 ein Verlagsgutachten, das den Druckgenehmigungsakten beiliegt. Freimütig beschreibt er das Werk als einen Beitrag in der literarischen Selbstfindung des Autors und scheut sich nicht, den Protagonisten Anicet - oft als Alter Ego Aragons gelesen

30. - Ebd., S. 74.

31. - Vor diesem Hintergrund ist es wichtig zu erwähnen, dass Aragon schon in der Zeit des sozialistischen Realismus von einigen Gutachtern in der DDR vorgeworfen wurde, eine zu große Verspieltheit an den Tag zu legen. Das lässt in gewisser Weise die Radikalität der ,Wende“ von 1962 fragwürdig erscheinen. Vgl. Bundesarchiv, Akte DR 1/3941, pag. 184.

32. - Bundesarchiv, Akte DR 1/2366, pag. 104. 
- zu einem frühen Vorläufer von „Camus’ Fremden“ zu erklären ${ }^{33}$. Auch Schewe geht davon aus, dass das Werk nur für einen kleinen Kreis „speziell Gebildeter und Interessierter“ von Bedeutung sei und plädiert somit für eine forschungsrelevante Veröffentlichung ${ }^{34}$. Auch wenn diese Argumentationsstrategie wenig mit der tatsächlichen Forschungstätigkeit zu tun hat, nimmt sie möglichen Ängsten vor einer breiten Wirkung, wie sie im Hauptamt für Verlage und Buchhandel bestanden haben mögen, im Vorfeld den Wind aus den Segeln. Ganz in diesem Sinne spricht Schewe davon, dass der Surrealismus endgültig Geschichte „ohne erkennbaren Aktualitätsbezug" geworden sei ${ }^{35}$. Zudem ist es ihm wichtig, dass die Veröffentlichung im Rahmen der Werkausgabe geplant ist, denn in einer Werkausgabe gilt der Anspruch, repräsentativ zu sein und man müsse somit mindestens ein Frühwerk publizieren. Schewe zeigt zudem geschickt Kontinuitäten auf: „Tatsächlich wäre es absurd, den surrealistischen Dichter von der Résistance trennen zu wollen"36". Das verbindende Element sei gerade das „Bewußtsein der Revolte ${ }^{37 \% “ . ~}$ 1976 wird der Antrag auf Druckgenehmigung von Volk und Welt eingereicht mit dem schriftlichen Vermerk auf dem Titelbogen des Formulars: „Kulturpolitisch klug angelegtes Nachwort dürfte auch die letzten Zweifel an der Veröffentlichung [...] ausräumen ${ }^{38 “ . ~ E s ~ i s t ~ w i e d e r u m ~}$ Schewe, der ein ausführliches, abgewogenes Nachwort verfasst, in dem der Surrealismus noch einmal als die Vorstufe in der dialektischen Entwicklung zum sozialistischen Realismus beschrieben wird. Doch es braucht tatsächlich noch ein Jahr und ein weiteres Gutachten von der Lektorin Georgina Baum, bis der Antrag genehmigt wird. Baum betont - und dies wird sich für alle weiteren Veröffentlichungen des Frühwerkes als die maßgebliche Argumentation erweisen - den Unterhaltungswert und den Spaß an der Lektüre ${ }^{39}$. Dabei wird Aragons dichterisches Verständnis des Spiels ganz neu und völlig entpolitisiert betrachtet. Weder die negative Verspieltheit als Argument der Surrealismusgegner, noch die respektgebietende Intellektualität von Person und Werk sind hier zentral, sondern der Genuss des ,merveilleux'. Es scheint, als habe es diese neue Auffassung nun möglich gemacht, in verhältnismäßig schneller Abfolge das restliche Frühwerk zu publizieren, ohne dass es noch der Belehrung eines wissenschaftlichen Nachwortes bedurft hätte.

33. - Bundesarchiv, Akte DR 1/2363, pag. 152.

34. - Ebd., S. 154.

35. - Ebd., s.o.

36. - Ebd., s.o.

37. - Ebd., s.o.

38. - Bundesarchiv, Akte DR 1/2363, pag. 139.

39. - Vgl. Bundesarchiv, Akte DR 1/2363, pag. 147. 


\section{Schlussbemerkungen}

An dieser vielschichtigen, keineswegs kohärenten Entwicklung des Rezeptionsverhaltens gegenüber Aragon, zeigt sich das Bestreben, den Vorzeigeautor nicht an den Westen zu verlieren und seine symbolische Bedeutung für eine spezifisch sozialistische Moderne in der Literatur weiterhin zu akzentuieren. Während es zu Beginn der Rezeption noch um die Findung einer literarischen Tradition der DDR geht, in der man weniger geneigt ist, ideologische Abweichungen hinzunehmen, gewinnen gegen Ende - auch dank differenzierterer Beschreibungskategorien immer mehr die literarischen Qualitäten an Bedeutung, selbst wenn dies eine Trivialisierung ehemals ideologischer Schützengräben zur Folge hat. So kann Schewe 1985 schreiben: „Täuschungen und Zerstörung traditioneller Lesererwartungen [...] gehören heute zum Allgemeingut moderner Literaturen ${ }^{40 " .}$.

Eine zukünftige Auseinandersetzung mit diesem Thema muss versuchen, ein vollständiges Abbild der Rezeption darzulegen, woran sich auch die Funktionsweise der Rezeptionsmechanismen in der DDR analysieren ließe. Gerade Louis Aragon ist für diese Analyse ein denkbar geeigneter Gegenstand. Denn die Verwischung von Grenzen ist bei ihm schon dadurch bedingt, dass sich in seinem Bild gleichzeitig der Parteifunktionär, der Literaturtheoretiker und der Autor spiegeln.

40. - Bundesarchiv, Akte DR 1/2385, pag. 52. 